BL/BLI Combinations

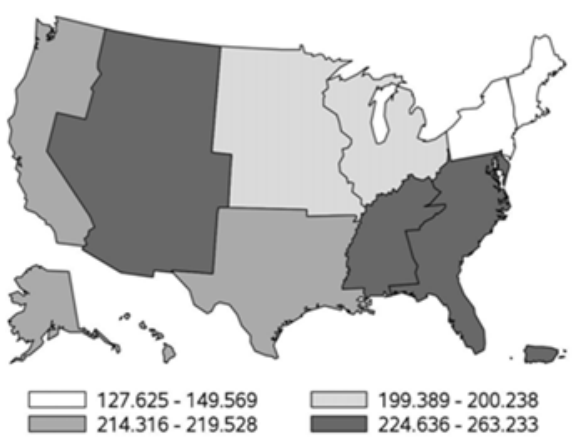

Glycopeptides

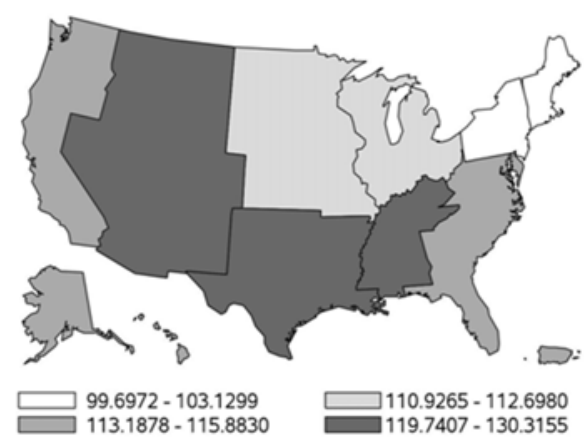

use was frequently associated with facilities likely to have robust antibiotic stewardship programs - those with teaching status and larger bed size. Further research to understand other reasons for regional differences in antibiotic use such as different rates of resistance is needed.

Funding: This work was supported by Funding: from the Agency for Healthcare Research and Quality (AHRQ) (R01-HS026205 to A.D.H.).

Disclosures: None

Doi:10.1017/ice.2020.534

\section{Preserntation Type:}

Oral Presentation

Surgical Antibiotic Prophylaxis in Hysterectomy: Is Cefazolin Still the Best?

Alyssa Valentyne, University of Wisconsin School of Medicine and Public Health; Fauzia Osman, University of Wisconsin School of Medicine and Public Health, Department of Medicine; Ahmed Al-Niaimi, University of Wisconsin School of Medicine and Public Health Nasia Safdar, University of Wisconsin, Madison; Aurora Pop-Vicas, University of Wisconsin School of Medicine and Public Health

Background: Prior studies suggest that cefazolin, widely used for antibiotic prophylaxis in hysterectomy, might not prevent surgical site infections (SSIs) as well as antibiotics with a broader antianaerobic antimicrobial spectrum. We compared the effectiveness of cefazolin versus antibiotic regimens with a broader antimicrobial spectrum in a $\geq 500$-bed regional referral center. Methods: Study design: retrospective cohort. Population and setting: patients $\geq 18$ years old who underwent hysterectomy between 1998 and 2018 at

\section{3rd/4th Gen. Cephalosporins}

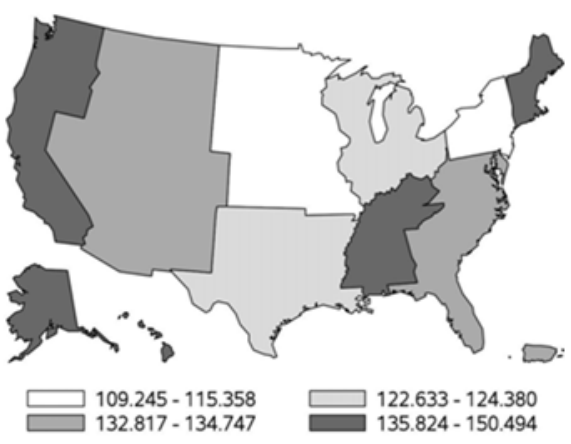

Carbapenems

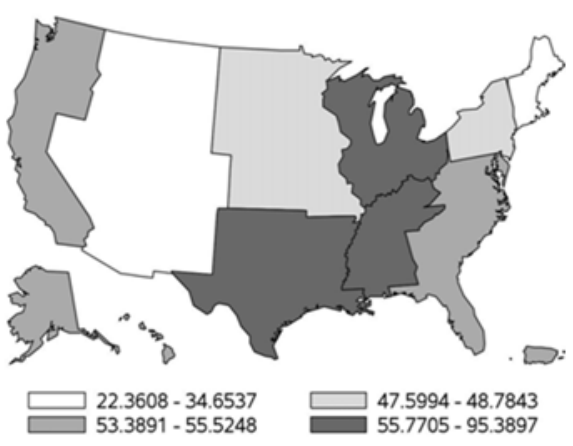

the University of Wisconsin Hospital. Analysis: propensity score matching with a caliper of 0.2 to select controls for cefazolin treatment, matching on: age, body mass index (BMI), diabetes, length of stay, duration of surgery, and preoperative renal function. We conducted a crude SSI incidence analysis and adjusted for additional covariates (malignancy, intraoperative temperature, and preoperative glucose level) using a Cox proportional hazards model. All analyses were conducted using STATA SE v15 software. Results: We had 4,087 hysterectomy patients, with 123 SSIs (3\%). Among these SSIs, $46 \%, 11 \%$, and $42 \%$ were superficial, deep, and organ-space, respectively. Malignancies were present in $83 \%$ of SSI patients, with $30 \%$ being ovarian cancer. Risk factors for SSI in the unmatched sample multivariable analysis (MV) were length of stay (aHR, 1.1; 95\% CI, $1.05-1.1 ; P<.001$ ), duration of surgery (aHR, $1.2 ; 95 \% \mathrm{CI}, 1.1-1.32 ; P$ $<.001$ ), and BMI (aHR, 1.04; 95\% CI, 1.02-1.06; $P<.001$ ). After propensity matching, 2,282 hysterectomies remained. In the crude incidence analysis, cefazolin (IR, 6.0) had a protective SSI effect compared to cefoxitin (IR, 7.1), ciprofloxacin/metronidazole (IR, 27.2), clindamycin/gentamicin (IR, 14.1), any antianaerobic regimen (IR, 8.0), and regimens not guideline recommended (IR, 11.7). In our MV analyses of cefazolin versus comparator antibiotic regimens, we found that hypothermia was consistently associated with a higher SSI risk $(P \leq .03)$. Receipt of a $\beta$-lactam antibiotic regimen was associated with a significantly lower SSI risk (aHR, 0.31 ; 95\% CI, $0.11-$ $0.89, P=.03$ ), but cefazolin's protective SSI effect was no longer statistically significant. Conclusions: We found that cefazolin had a lower SSI risk compared to other antibiotic regimens, including those with better antianaerobic spectrum, in our tertiary-care hospital's 11-year high-risk cohort. Our analysis suggests that maintaining intraoperative normothermia and administering $\beta$-lactam antibiotic prophylaxis are important modifiable risk factors for SSI prevention. 
Funding: None

Disclosures: None

Doi:10.1017/ice.2020.535

\section{Presentation Type:}

Oral Presentation

Transmission of Listeriosis in a Neonatal Intensive Care Unit Supported by Whole-Genome Sequencing

Janice Kim, California Dept. of Public Health; Hilary Rosen, California Department of Public Health; Kristen Angel, County of San Diego Health and Human Services Agency; Azarnoush Maroufi, San Diego County Health and Human Services Agency; Samantha Tweeten, San Diego County Health and Human Services Agency; Jacqueline Lui, California Department of Public Health; John Crandall, California Department of Public Health; Tracy Lanier, California Department of Public Health; Jane Siegel, California Department of Public Health, Richmond, CA; Akiko Kimura, California Department of Public Health

Background: Listeriosis is a rare but serious infectious disease caused by Listeria monocytogenes (LM) and predominantly transmitted through contaminated food. Moreover, 15\% of listeriosis cases in the United States are pregnancy associated; nosocomial neonatal transmission in hospitals is extremely rare. In July 2018, the California Department of Public Health (CDPH) was notified of 4 patients, a mother-neonate pair and twin neonates, with listeriosis at the same hospital. The CDPH and San Diego County Health and Human Services Agency initiated an investigation to determine transmission and prevent additional infections. Methods: We reviewed medical records of the neonates and their mothers, interviewed the mothers with a detailed food exposure questionnaire, interviewed healthcare personnel (HCP), and performed an infection control assessment of the neonatal intensive care unit (NICU). CDPH performed whole-genome sequencing (WGS) on LM isolates that were then analyzed by whole-genome multilocus sequence typing (wgMLST) by the Centers for Diseases Control and Prevention (CDC) to assess relatedness in PulseNet, a public health laboratory database. The CDC also performed testing for LM on formalin-fixed placentas from the mother of the twins. Results: During a 1-week period, 4 patients with LM were identified at the hospital. A mother was admitted at 31 weeks gestation with acute abdominal and back pain that progressed with precipitous vaginal delivery and postpartum sepsis. Her neonate was resuscitated, transported to the NICU, underwent a sepsis evaluation, received antibiotics, and was transferred to another hospital within 6 hours. Maternal blood, placenta, and neonatal blood cultures grew LM. Twin neonates, born to an asymptomatic mother and present in the NICU during the index neonate's stay, developed acute infection 4 and 6 days after the index neonate's transfer; blood cultures confirmed LM. The LM isolates from the 4 patients were indistinguishable by wgMLST and were not related to other PulseNet isolates. LM was not detected in the twin placentas. There were no common food exposures between the mothers. At least 1 common HCP cared for all 3 neonates. Infection control lapses included lack of proper hand hygiene during the index neonate's resuscitation and potentially after cleaning and disinfection of the neonate's incubator. Conclusions: This report provides supportive evidence that nosocomial transmission of LM can occur during a brief NICU stay due to lapses in infection control practices. Strict adherence to standard precautions in the delivery room and NICU is imperative to prevent cross transmission.
Disclosures: None

Funding: None

Doi:10.1017/ice.2020.536

Presentation Type:

Oral Presentation

Trends in Hospital Onset Clostridioides difficile Infection Incidence, National Healthcare Safety Network, 2010-2018

Yi Mu, Centers for Disease Control and Prevention; Margaret Dudeck, Centers for Disease Control and Prevention; Karen Jones, CACI; Qunna Li, Centers for Disease Control and Prevention; Minn Soe, Centers for Disease Control and Prevention; Allan Nkwata, Centers for Disease Control and Prevention; Jonathan Edwards, Centers for Disease Control and Prevention

Background: Clostridioides difficile infection (CDI) is one of the most common laboratory-identified (LabID) healthcare-associated events reported to the National Healthcare Safety Network (NHSN). CDI prevention remains a national priority, and efforts to reduce infection burden and improve antibiotic stewardship continue to expand across the healthcare spectrum. Beginning in 2013, the Centers for Medicare and Medicaid Services (CMS) required acute-care hospitals participating in CMS' Inpatient Quality Reporting program to report CDI LabID data to NHSN and, in 2015, extended this reporting requirement to emergency departments (ED) and 24-hour observation units. To assess national progress, we evaluated changes in hospital onset CDI (HO-CDI) incidence during 2010-2018. Methods: Cases of HOCDI were reported to NHSN by hospitals using the NHSN's LabID criteria. Generalized linear mixed-effects modeling was used to assess trends of HO-CDI by treating the hospital as a random intercept to account for the correlation of the repeated responses over time. The data were summarized at the quarterly level, the main effect was time, and the covariates of interest were the following: CDI test type, inpatient community-onset (CO) infection rate, hospital type, average length of stay, medical school affiliation, number of beds, number of ICU beds, number of infection control professionals, presence of an ED or observation unit, and an indicator for 2015 to account for CDI protocol changes that required hospitals to conduct surveillance in both inpatient and ED or observation unit setting. Results: During 2010-2013, the number of hospitals reporting CDI increased and then stabilized after 2013 (Table 1). Crude HO-CDI rates decreased over time, except for an increase in 2015 and steeper reduction thereafter. (Table 2). During 2010-2014, the adjusted quarterly rate of change was $-0.45 \%$ ( $95 \% \mathrm{CI},-0.57 \%$ to $-0.33 \% ; P<.0001)$. The rate of reduction was smaller in 2010-2014 compared to those of 2015-2018

\section{Table 1.}

Table 1. Annual crude healthcare facility-onset Clostridioides difficile infection (HO-CDI) rates, 2010-2018

\begin{tabular}{lrrrr}
\hline Year & No. of facilities & No. HO events & No. of patient days & HO Rate / 1,000 \\
\hline 2010 & 618 & 19,870 & $27,395,881$ & 0.73 \\
2011 & 777 & 26,103 & $35,301,030$ & 0.74 \\
2012 & 1,462 & 40,365 & $55,376,026$ & 0.73 \\
2013 & 3,492 & 98,673 & $144,621,429$ & 0.68 \\
2014 & 3,502 & 99,831 & $145,208,862$ & 0.69 \\
2015 & 3,512 & 100,464 & $140,196,226$ & 0.72 \\
2016 & 3,536 & 95,371 & $141,226,760$ & 0.68 \\
2017 & 3,608 & 81,854 & $142,283,723$ & 0.58 \\
2018 & 3,588 & 69,281 & $143,004,833$ & 0.48 \\
\hline
\end{tabular}

